\title{
CORRECTION OF NAVIGATIONAL INFORMATION SUPPLIED TO BIOMIMETIC AUTONOMOUS UNDERWATER VEHICLE
}

Tomasz Praczyk

Polish Naval Academy, Gdynia, Poland

\begin{abstract}
In order to autonomously transfer from one point of the environment to the other, Autonomous Underwater Vehicles $(A U V)$ need a navigational system. While navigating underwater the vehicles usually use a dead reckoning method which calculates vehicle movement on the basis of the information about velocity (sometimes also acceleration) and course (heading) provided by on-board devicesl ike Doppler Velocity Logs and Fibre Optical Gyroscopes. Due to inaccuracies of the devices and the influence of environmental forces, the position generated by the dead reckoning navigational system (DRNS) is not free from errors, moreover the errors grow exponentially in time. The problem becomes even more serious when we deal with small AUVs which do not have any speedometer on board and whose course measurement device is inaccurate. To improve indications of the DRNS the vehicle can emerge onto the surface from time to time, record its GPS position, and measure position error which can be further used to estimate environmental influence and inaccuracies caused by mechanisms of the vehicle. This paper reports simulation tests which were performed to determine the most effective method for correction of DRNS designed for a real Biomimetic AUV.
\end{abstract}

Keywords: underwater navigation, odometry, underwater vehicle

\section{INTRODUCTION}

To make Autonomous Underwater Vehicle (AUV) [15] capable of acting without an external support it has to be equipped with systems which ensure its operational independence. To decide about actions which has to be taken to achieve a desired goal, the vehicle has to be supplied with information describing the state of the vehicle and its surrounding. The fundamental information required for safe underwater navigation is that about vehicle position.

To acquire such information in a fully autonomous mode, a dead reckoning is usually used by AUV. Knowing a starting position of the vehicle, its orientation and velocity, the dead reckoning navigation can estimate motion of the vehicle, and in consequence, its position. To measure orientation, compasses, Inertial Navigational Systems (INS), and Fibre Optical Gyroscopes (FOG) can be applied. In turn, velocity in the underwater environment is measured by using logs. There are different types of logs, namely, mechanical, electromagnetic, pressure, and Doppler logs which differ in accuracy and size. Electromagnetic ones seem to be the smallest in size, however, they are rather inapplicable to lower speeds of underwater vehicles. The same applies to mechanical logs. The Doppler logs are very precise, even for low speeds, moreover they measure velocity with respect to the seabed, which means that they take sea current into 
account in their measurements. Their drawback is, however, large cost and size which make them impractical in use on board of small vehicles.

An alternative solution for logs, especially for small vehicles like those presented in Fig. 1, is odometry. It estimates momentary velocity of the vehicle on the basis of the information from its control system. When the vehicle has a limited number of motion modes (or motion patterns), e.g. fast sharp turn, slow sharp turn, slow gentle turn, slow move ahead, submerge vertically, average speed of the vehicle in each mode may be measured and then used for position calculation.

a)

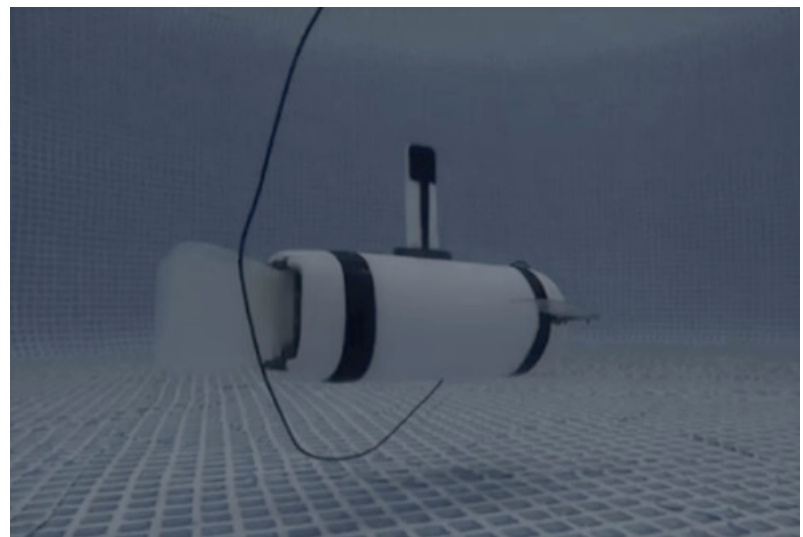

b)

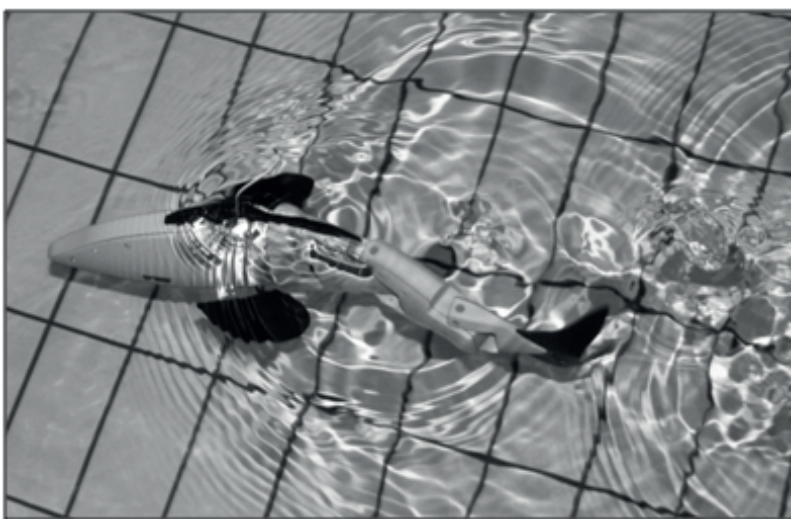

Fig. 1 Small biomimetic vehicles, (a) - AUV called CyberSeal, (b) - Remotely Operated Vehicle called CyberFish [11]

All the above mentioned solutions, regardless of the applied device technology, produce a position error which depends on three factors:

- the accuracy of the orientation and velocity (provided by the above mentioned navigational devices),

- vehicle controllers (velocity, depth and course controller) whose task is to maintain desired vehicle parameters, and

- environmental factors, of a random nature, e.g. the sea current. To improve the accuracy of position produced by a dead reckoning navigational system (DRNS), we can try to estimate all the above specified factors and take them into account while calculating vehicle position. To this end, the vehicle can emerge onto the surface from time to time, record its GPS position, determine position error, and finally use it to estimate all forces which affect the error. a)

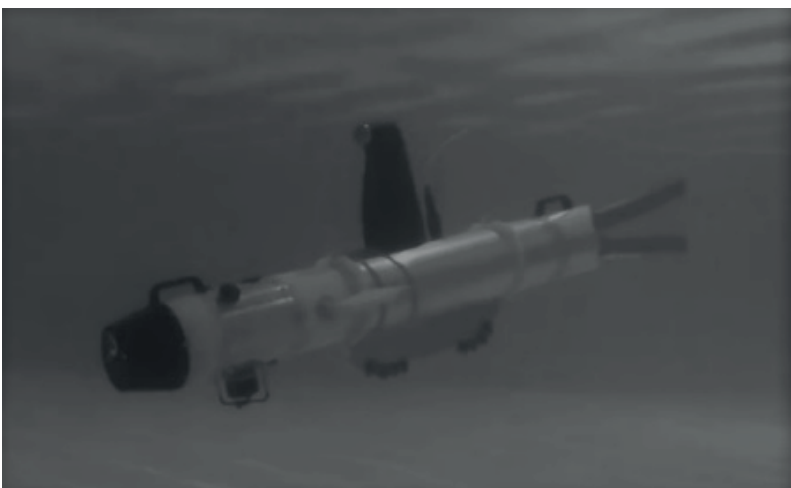

b)

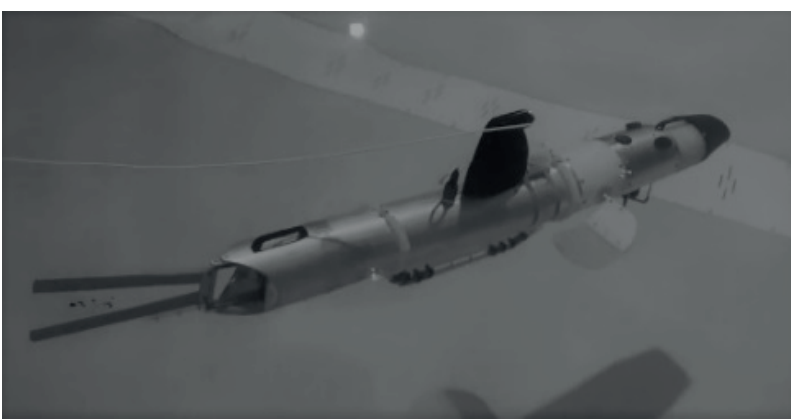

Fig. 2 BAUV used in experiments in the pool

To verify usefulness of this approach for the Biomimetic Autonomous Underwater Vehicle (BAUV) (see Fig. 2) being designed within the project titled "Autonomous underwater vehicles with silent undulating propulsion for underwater ISR", and financed by Polish National Centre of Research and Development $[13,14,16,17]$, simulation tests were carried out whose results are presented in this paper. Application of a correction system for BAUV DRNS appeared to be necessary during initial tests in a $25 \mathrm{~m}$ swimming pool. The vehicle is equipped with only two navigational devices, i.e. a digital compass to measure course and pressure sensor used to sense depth. There is no device to measure speed which is determined by means of odometry. The consequence is that accuracy of the DRNS is inadequate with respect to tasks imposed on the BAUV. Example wrong locations of the BAUV in the swimming pool, determined by DRNS are depicted in Fig. 3, the straight lines indicate simultaneously a desired path of the vehicle and left and upper edges of the pool. In spite of the fact that the vehicle is inside the pool, the DRNS indicates something else.

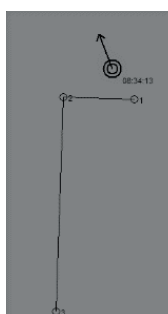

a)

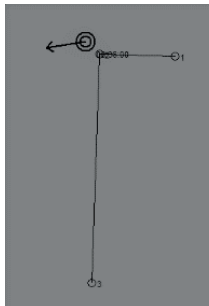

b)

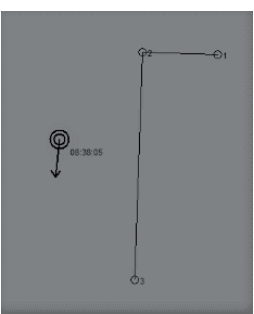

c)
Fig. 3 Example location of BAUV during experiments in the pool 
The rest of the paper is organized as follows: Section 2 is a short review of the presented work, Section 3 is a description of position correction method applied to the simulations, Section 4 is a report from the experiments, and the final section is a summary.

\section{CONSIDERED WORK}

According to $[8,10]$ underwater navigation has generally two main approaches, namely: dead reckoning, and acoustic. In the dead reckoning navigation $[1,3,12]$ we deal with three main components, i.e. sensors, kinematic model of underwater vehicle, and vehicle state estimation method. The estimation method, typically, family of Extended Kalman Filters (EKF) [5] or Unscented Kalman Filters (UKF) [7,18], but also Particle Filters [2,4], is supplied with the vehicle model and sensor outputs (observations) and produces estimations of vehicle state vector including at least position of the vehicle in a chosen coordinate system. The sensors provide information about orientation of the vehicle (INSs, digital compasses, FOGs), depth (pressure sensors), velocities (logs) and sometimes also accelerations (INSs). The more information from the sensors and the more accurate it is, the more accurate the estimations. Of course, the same applies to kinematic model of the vehicle.

When estimations are performed basing on reliable information about spatial orientation and velocity acquired e.g. from optical gyro and Doppler log, moreover, when the vehicle has classical torpedo-shape construction with one rear screw propeller which is equivalent to presence of reliable models of such vehicles, and the sea current is estimated, e.g. basing on historical data or satellite photos, then results of dead reckoning approach are usually satisfactory. However the application of the above discussed approach seems to be impossible in case when the vehicle cannot directly sense its velocity, information about accelerations is available but seriously disturbed (hence it is practically useless at least to accurately estimate velocity), information about the spatial orientation is less accurate than that produced by optical gyros, and moreover, the vehicle itself is highly complicated in terms of construction which results in lack of its reliable model. Unfortunately, this is exactly what we are dealing with in the case of the BAUV.

A separate approach in the dead reckoning navigation is application of visual information about surrounding environment as observations to estimation methods $[6,19,20]$. However, this approach known as Simultaneous Mapping and Localization (SLAM) requires the vehicle to move close the seabed, and moreover, to have special equipmentlike sonar or, rarely, a camera.

As mentioned above, the other approach in the underwater navigation is an acoustic stream (Long Baseline, Ultrashort Baseline, and Short Baseline systems - LBL, USBL and SBL) which is generally based on location in relation to sources of an acoustic signal of a known position. The main drawback of this approach is the need to have an additional infrastructure (except of the vehicle itself) with the effect that it can be only used locally in areas which allow to deploy the system. Of course, the other issue is preparation of the area to work, which requires a time.

\section{CORRECTION OF DRNS}

\section{FUNDAMENTALS}

DRNS of the BAUV is a navigation system with a school kinematic model of the vehicle in the form of $S=V t$, where: $S$ - displacement in the horizontal plane, $V$ - a velocity, and $t$ - a time interval. The model is supplied with vehicle progressive speed provided by the odometry, course obtained from a digital compass, and additionally, a vehicle depth - from a pressure sensor. The speed and the course are used to calculate the horizontal displacement and the pressure sensor determines the depth. Simplicity of the vehicle model applied in DRNS results from difficulty in defining more accurate models of such vehicles as the BAUV. It has three independent propellers in the form of fins which ,when course and depth controllers are activated, can work in different directions and with a different force, with the effect that e.g. trim (pitch) of the vehicle does not generally reflect change of depth.

The consequence of such very simple vehicle model and its poor equipment is that to apply the dead reckoning approach outlined in the previous section is impossible. Effective usage of the state estimation methods like EKF or UKF requires to confront outputs of the model and sensors. In the simplest case, vehicle orientation along with progressive speed is used in the model to calculate $z$ - coordinate of the vehicle (coordinate towards Earth). At the same time, $z$ - coordinate is also measured by a pressure sensor with the effect that both $z$-values can be confronted with each other and the whole vehicle state vector produced by the model can be recalculated and improved.

Unfortunately, in the case of the BAUV, we do not have such comfort. The cause is that, as mentioned above, the orientation of the BAUV does not reflect change of depth. In consequence, we cannot estimate $z$ - coordinate based on the measured orientation and odometric speed, compare it with a measured value of $z$, and finally, improve vehicle position estimated by the model.

However, to improve accuracy of DRNS we can periodically resurface the BAUV to get precise GPS position, roughly estimate all internal and external forces having influence on the vehicle motion and use the estimates (along with other available vehicle state parameters) for position calculation. At the very start of a vehicle mission, DRNS relies only on odometric speed, orientation measured by compass and the depth fixed by a pressure sensor. Then, a correction procedure is performed to estimate the above mentioned forces and use them in calculations of vehicle coordinates $x y$. The depth 
of the vehicle is still fixed by the pressure sensor only. Details of the correction procedure are given in the following section.

\section{FORMULATION}

The correction method proposed in this paper and described below is based on the assumption that the correction procedure $(\mathrm{CP})$ is always carried out during BAUV mission. There is no separate CP performed in advance, the BAUV runs the mission and the $\mathrm{CP}$ is its integral part.

A perfect situation for the CP would be to perform it in two distinct stages, first, in the area without any influence of the environment or at most with a slight influence, and then in the vehicle operational area. In this case, the objective of the first stage would be to determine inaccuracies resulting from the vehicle itself, whereas the second stage would be dedicated exclusively to estimate influence of environmental factors like the sea current. Unfortunately, a number of tests performed by a project team showed that even lakes which at first glance should be filled with still water, are inadequate for the first stage of CP, as the environmental factors, e.g. the wind, may cause water to move, making the objective of the first stage unattainable.

Moreover, since the vehicle can be used in different regions of the world with different salinity of water, we cannot assume that to adjust its buoyancy to the future operational area would be possible in advance. Since each change in vehicle construction (also in its buoyancy) causes changes in vehicle behaviour, which affect in turn the navigation, a separate $\mathrm{CP}$ considering only the vehicle, even in a lake with slight environmental influence, is not a solution which can ensure DRNS adequate accuracy in various operational areas.

In consequence, as mentioned above, the assumption is that the CP is performed just during the mission. The vehicle should emerge from time to time onto the surface for recording GPS position, calculating position error, and then fixing the correction. At the very start of the mission, the influence of both factors on DRNS inaccuracies should be only once estimated, then only environmental factors should be periodically determined, and, as inaccuracies affected by the vehicle are assumed to be invariable, they can be fixed only once. Since estimation of only one factor affecting DRNS inaccuracies is a trivial problem, only CP carried out at the beginning of the mission is considered in this paper. To this end, two different solutions are presented, say : CP1 and $\mathrm{CP} 2$, the first one is when CP cannot be a separate part of the mission, hence it has to be performed in the meantime, whereas the second one is when the beginning of the mission is devoted especially to $\mathrm{CP}$, then the main part of the mission starts immediately after realization of the procedure.

But let us start with the formulation of vehicle motion with velocity $V_{a}$ in $x$ - and $y$-direction in the time $T$ :

$$
S_{x}=\int_{0}^{T}\left(V_{a}^{x}(t)+V_{e}^{x}(t)\right) d t+\int_{0}^{T} V_{c}^{x}(t) d t+\int_{0}^{T} V_{r}^{x}(t) d t
$$

$S_{y}=\int_{0}^{T}\left(V_{a}^{y}(t)+V_{e}^{y}(t)\right) d t+\int_{0}^{T} V_{c}^{y}(t) d t+\int_{0}^{T} V_{r}^{y}(t) d t$

where:

$S_{x}, S_{y}$-displacements of vehicle in $x$ - and $y$ - direction in the time $T$, or $x$ - and $y$-coordinates of vehicle originating from the noint $x=0, y=0$;

$V_{a}^{x}, V_{a}^{y}-x$ - and $y$-components of the vehicle velocity $V_{a}$ measured by vehicle devices (direction) and odometry (magnitude);

$V_{\theta}^{x} \cdot V_{\theta}^{y}-x$ - and $y$ - components of the vehicle velocity error $V_{\theta}$;

$V_{c}^{x}, V_{c}^{y}-x$ - and $y$ - components of the sea current velocity $V_{f}$;

$V_{r}^{x}, V_{r}^{f}-x$-and $y$-components of the random velocity $V_{r}$

In Eq. (1) and (2), $V_{r}$ is assumed to be very small compared to the remaining velocities and, moreover, its direction is random which means that in the long term the influence of $V_{r}$ on the vehicle can be neglected - as the momentary velocities $V_{r}$ are assumed to be compensated in the long term. In effect, Eq. (1), (2) can be simplified to the following form:

$$
\begin{aligned}
& S_{x}=\int_{0}^{T}\left(V_{a}^{x}(t)+V_{e}^{x}(t)\right) d t+\int_{0}^{T} V_{c}^{x}(t) d t \\
& S_{y}=\int_{0}^{T}\left(V_{a}^{y}(t)+V_{e}^{y}(t)\right) d t+\int_{0}^{T} V_{c}^{y}(t) d t
\end{aligned}
$$

Moreover, if we assume that the move of the vehicle along a desired trajectory takes place between successive way-points with application of course and depth controllers, and the sea current is invariable at a short distance, Eq. (3), (4) for the vehicle moving between two neighboring way-points with the course angle equal to $\alpha$, can be rewritten as follows:

$$
S_{y}=S_{x}=\left(V_{a}^{x}+V_{\varepsilon}^{x}+V_{c}^{x}\right) T
$$

$$
\left(V_{a}^{x} \tan (\psi(\alpha))+V_{e}^{x} \tan (\psi(\alpha+\beta))+V_{c}^{x}\right) T
$$

where:

$\beta$-deviation from the course $\alpha$;

$$
\psi(\theta)=\left\{\begin{array}{c}
90-\theta \text { if } \theta \in\langle 0,180\rangle \\
270-\theta \text { otherwise }
\end{array} \quad \theta \neq 90, \theta \neq 270\right.
$$

Since the BAUV is not equipped with velocity measurement devices, moreover, we cannot also measure other velocities, we assume that all the velocities are constant in all calculations. In this case, $V_{a}$ is a velocity in direction indicated by the course $\alpha$ and maintained by BAUV course controller. As previously, $\left|V_{a}\right|$ is determined by odometry and it corresponds to some settings of vehicle drive. In this case vehicle compass is used by the controller to maintain the course. Other velocities are also assumed to be constant, $V_{c}$ is the velocity which is caused mainly by the sea current, at a short distance between two neighboring way-points it has a constant direction and value, 
whereas $V_{\theta}$ is constant in terms of value and deviated from $\alpha$ by a constant angle $\beta$, and it corresponds to all the features of the vehicle ( like asymmetry of propellers, compass errors etc ) which can have impact on DRNS errors.

The function $\psi$ used in Eq.(6) reduces $\theta$ to the range $\langle 0,90) \$$ and simultaneously determines $V_{n}^{y}$ and $V_{\theta}^{y}$ with respect to $V_{a}^{x}$ and $V_{\theta}^{x}$. For example, if $\theta=60$, then $V_{a}^{x}$ and $V_{a}^{y}$ act in the same direction and $V_{a}^{y}=V_{a}^{x} \tan (30)$, whereas, if $\theta=120$, then $V_{a}^{y}=-V_{a}^{x} \tan (30)$.

Before the CP, the only values known to the DRNS is $V_{a}$ and $\alpha$. In consequence, vehicle motion is described as follows: $S_{x, 1}=V_{a}^{x} T, S_{y, 1}=V_{a}^{x} \tan (\psi(\alpha)) T$ (“1” means the first way-point). Once the BAUV emerges onto the surface, its GPS position can be recorded and DRNS position error can be calculated, that we denote as $\Delta S_{x, 1}=S_{x, G P S}-S_{x, 1}$ and $\Delta S_{y, 1}=S_{y, G P S}-S_{y, 1}$. Assuming that the only velocity which "pushes" the BAUV towards $\left(S_{x, 1}, S_{y, 1}\right)$ position is $V_{a}$, the remaining velocities are responsible for the error. In consequence, it is sufficient to fix $V_{\theta}, V_{c}$ and $\beta$ and take them into account when calculating the BAUV position to improve its accuracy. To this end, we have to solve the following system of equations:

$$
\begin{gathered}
\Delta S_{x, 1}=\left(V_{s, 1}^{x}+V_{c, 1}^{x}\right) T_{1} \\
\Delta S_{y, 1}=\left(V_{s, 1}^{x} \tan \left(\psi\left(\alpha_{1}+\beta\right)\right)+V_{c, 1}^{y}\right) T_{1} \\
\Delta S_{x, 2}=\left(V_{s, 2}^{x}+V_{c, 2}^{x}\right) T_{2} \\
\Delta S_{y, 2}=\left(V_{\theta, 2}^{x} \tan \left(\psi\left(\alpha_{2}+\beta\right)\right)+V_{c, 2}^{y}\right) T_{2}
\end{gathered}
$$

Moreover, as $\left|V_{\theta, 1}\right|=\left|V_{\theta, 2}\right|$ we can also write the following:

$\left(V_{\theta, 1}^{x}\right)^{2}+\left(V_{\theta, 1}^{x}\right)^{2} \tan ^{2}\left(\psi\left(\alpha_{1}+\beta\right)\right)=\left(V_{\theta, 2}^{x}\right)^{2}+\left(V_{\theta, 2}^{x}\right)^{2} \tan ^{2}\left(\psi\left(\alpha_{2}+\beta\right)\right)$

In both Eq. (7),(8) and Eq.(9),(10) which specify position error in two consecutive way-points, we deal with the velocity $V_{\theta}$ which does not change its magnitude but changes direction , and with the velocity $V_{c}$ which changes its magnitude and does not change direction.

a)

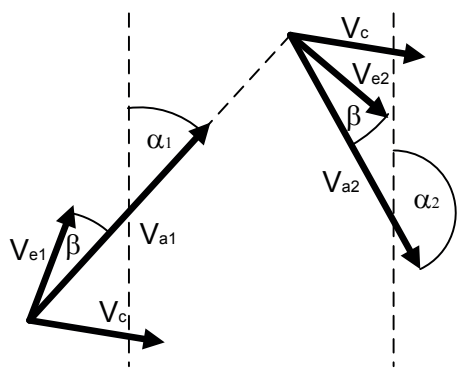

b)

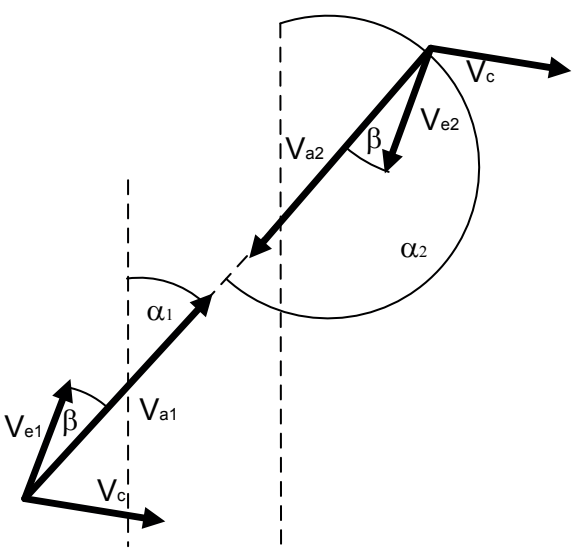

Fig. 4 Illustration of CP1 (a) and CP2 (b)

Direction of $V_{\theta}$ is determined by the vehicle course $\alpha$ and deviation $\beta$. If the course is different while navigating to both way-points, direction of $V_{e}$ is in effect also different, whereas the magnitude of $V_{e}$ is the same in both cases because settings of drive are assumed to be the same during voyage both to way-point No. 1 and 2.

In turn, $V_{c}$ is generally assumed to not change direction and magnitude, however, in order to take into account a different influence of the sea current on the vehicle hull depending on direction of vehicle course and direction of the current $t^{1}$, the angle $\gamma$ between vectors $V_{c}$ and $V_{a}$, and magnitudes of $V_{c}$ different for different courses $\alpha$ should be taken into consideration.

\section{CORRECTION PROCEDURE NO. 1 - CP1}

As mentioned above, the CP1 is based on the assumption that the procedure is performed simultaneously with other vehicle tasks, it does not have the vehicle at its disposal only. The consequence is lack of any limitations for the vehicle course when CP1 is performed in successive way-points (with one exception - see further). The other assumption made for CP1 is neglecting different sea current dynamic features for different courses of the vehicle. As a result, Eq.(7)(10) are simplified to the form containing $V_{c, 1}^{x}=V_{c, 2}^{x}$ and $V_{c, 1}^{y}=V_{c, 2}^{y}$.

To determine $V_{\theta}, V_{c}$ and $\beta$, the CP1 is performed in two phases. First, it calculates $V_{\theta}$ and $V_{c}$ with the assumption that $\beta=0$. For that purpose, the system of linear equations (7)-(10) is solved. To make it solvable, the above mentioned assumption regarding the courses has to be somewhat relaxed, namely, the courses to way-points No. 1 and 2 cannot be the same and opposite. After the first phase, a simple gradient descent method is used for the following objective function to be minimized:

$$
F\left(V_{\theta, 1}^{x}, V_{\theta, 2}^{x}, V_{c}^{x}, V_{c}^{y}, \beta\right)=\sum_{i=1}^{5} \text { Error }_{i}
$$

\footnotetext{
1 The influence of the sea current on the vehicle hull is definitely more complex than the paper suggests. However, due to high complexity of the precise current model, for the CP purposes, it was reduced to the form described in the paper.
} 
where

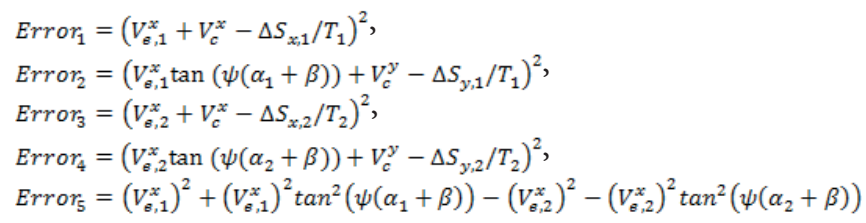

To increase the chance of finding a global optimum of the function (12), the optimization algorithm is repeated from many starting points which differ in value of $\beta$. Starting values for $V_{\theta}$ and $V_{c}$ do not change and they are solutions of the linear problem.

The result of the optimization procedure are values $\left|V_{\theta}\right|, \beta$, $V_{c}^{x}$ and $V_{c}^{y}$ which can be then used during vehicle navigation to improve DRNS indications.

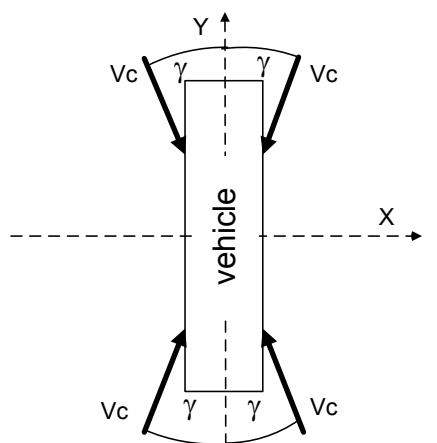

Fig. 5 The same influence of the see current on vehicle hull for angles $\gamma$, $180-\gamma, 180+\gamma$, and $360-\gamma$

\section{CORRECTION PROCEDURE NO. 2 - CP2}

In the case of CP2, the beginning of BAUV mission is devoted exclusively to $\mathrm{CP}$ which means that we can adjust operation of the vehicle to the CP. In CP2, it is done by moving the vehicle first along the course $\alpha_{1}=45$ and then along the opposite course $\alpha_{2}=225$. Thanks to that, to determine $V_{\theta}, V_{c}$ and $\beta$, it is enough to solve the following simple system of equations:

$$
\begin{gathered}
V_{\theta, 1}^{x}+V_{c}^{x}=\frac{\Delta S_{x, 1}}{T_{1}}=A \\
V_{\theta, 1}^{x} \tan \left(\psi\left(\alpha_{1}+\beta\right)\right)+V_{c}^{y}=\frac{\Delta S_{y, 1}}{T_{1}}=B \\
-V_{\theta, 1}^{x}+V_{c}^{x}=\frac{\Delta S_{x, 2}}{T_{2}}=C \\
-V_{\theta, 1}^{x} \tan \left(\psi\left(\alpha_{1}+\beta\right)\right)+V_{c}^{y}=\frac{\Delta S_{y, 2}}{T_{2}}=D
\end{gathered}
$$

In CP2 the same magnitude of $V_{c}$ in both directions of vehicle motion is also assumed. This assumption results from that the influence of the sea current on the vehicle hull is symmetrical with respect to $X$ - and $Y$-axes of the vehicle, i.e., it is the same e.g. for the angles of 10, 170, 190 and 350 deg - see Fig. 5.

The solution of Eq. (13)-(16) looks as follows:

$$
\begin{gathered}
V_{\theta, 1}^{x}=\frac{A-C}{2} \\
V_{c}^{x}=A-\frac{A-C}{2} \\
\beta=\arctan \left(\frac{B-D}{A-C}\right)-\alpha_{1} \\
V_{c}^{y}=B-\left(\frac{A-C}{2}\right) \tan \left(\arctan \left(\frac{B-D}{A-C}\right)\right)
\end{gathered}
$$

Of course, knowing $V_{\theta, 1}^{x}$ and $B$ we can calculate

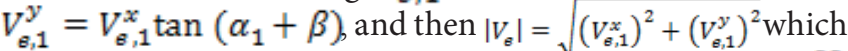
, along with $\beta$, is necessary to determine the influence of $V_{\theta}$ on motion of the vehicle in each point of the voyage.

Unfortunately, the velocities $V_{c}^{x}$ and $V_{c}^{y}$ calculated according to Eq. (13), (14) can be only used for the course $\alpha_{1}$ and the opposite course, for other vehicle courses they have to be recalculated. To this end, a simplified model $\xi\left(V_{c, \alpha_{1}}^{x}, V_{c, \alpha_{1}}^{y}, \alpha_{1}, \alpha_{2}\right)$ of the sea current influence on the vehicle hull is used. It produces the magnitude $\left|V_{c, \alpha_{2}}\right|$ for any vehicle course $\alpha_{2}$. Since direction of the sea current is assumed to not change $V_{c, \alpha_{2}}^{x}$ and $V_{c, \alpha_{2}}^{y}$, it can be then calculated basing on $V_{c, \alpha_{1}}^{x}, V_{c, \alpha_{1}}^{y}$ and $\left|V_{c, \alpha_{n}}\right|$.

The model $\xi$ is based on the function $\varrho(\gamma)$ which produces the current magnitude $\left|V_{c}\right|$ for any angle $\gamma$, i.e. the angle between vehicle course and direction of the sea current. The value generated by $\varrho$, according to the assumptions made for $\mathrm{CP} 2$, is symmetrical with respect to $X$ - and $Y$ - axes of the vehicle, and it corresponds to velocity of the vehicle "Gluptak" (see Fig. 6) equal to $1 \mathrm{~m} / \mathrm{s}$ and velocity of the sea current equal to $0.4 \mathrm{~m} / \mathrm{s}$. The vehicle "Gluptak" is a torpedo-shape ROV (Remotely Operated Vehicle) of the size and shape similar to the BAUV.

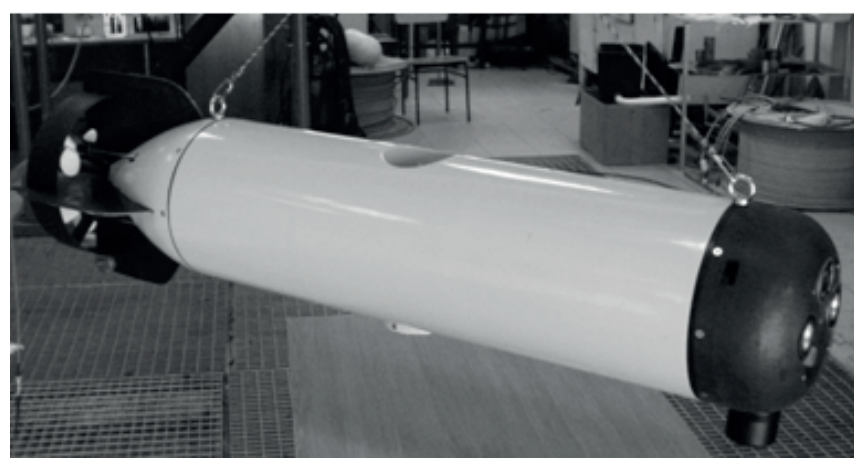

Fig. 6 ROV “Gluptak" [9,21] 
To determine the value $\left|V_{c}\right|$ for other velocities, in the model $\xi$ it is assumed that $\frac{\rho\left(\gamma_{1}\right)}{o\left(\gamma_{2}\right)}$ is constant regardless of vehicle and sea current velocities. The consequence of the above given assumption is possibility of calculating $\left|V_{c, \alpha_{2}}\right|$ for any vehicle course $\alpha_{2}$ in the following way:

$\left|V_{c, \alpha_{2}}\right|=\xi\left(V_{c, \alpha_{1}}^{x}, V_{c, \alpha_{1}}^{y}, \alpha_{1}, \alpha_{2}\right)=\left|V_{c, \alpha_{1}}\right| \frac{\varrho\left(\gamma_{2}\right)}{\varrho\left(\gamma_{1}\right)}$

where :

$\gamma_{i}=\operatorname{diffCourse}\left(\right.$ getCourse $\left.360\left(V_{c, \alpha_{i}}^{x}, V_{c, \alpha_{i}}^{y}\right), \alpha_{i}\right), i=1,2$, diffCourseis a function that gets difference between courses ranging $\langle 0,180\rangle \mathrm{deg}$, whereas function getCour se produces course based on $V^{x}, V^{y}$.

\section{CONSIDERING PRESENCE OF ENVIRONMENTAL FORCES WHEN NAVIGATING TO WAY-POINT}

When navigating to a way-point two different strategies can be used. The first one is simply to determine a direct course $v$ to the way-point and updating it periodically. This strategy is, however, impractical when the vehicle operates in the environment with a serious influence of the current and/or wind. In this case, the vehicle path to the way-point is very far from the optimum one, i.e. the path which leads the vehicle straight from the current vehicle position to the way-point.

The other strategy is to estimate all environmental forces which may have influence on the vehicle motion and to take them into account when determining the vehicle course $\alpha$ to the way-point. In the case of CP1, to calculate the course the following system of equations is solved ${ }^{2}$ :

$$
\begin{gathered}
\frac{V_{a}^{x}+v_{c, C P}^{x}}{V_{a}^{y}+V_{c, C P}^{y}}=\tan (\vartheta(v)) \\
\left(V_{a}^{x}\right)^{2}+\left(V_{a}^{y}\right)^{2}=\left|V_{a, C P}\right|
\end{gathered}
$$

where ;

$V_{a}^{x}, V_{a}^{y}-x$ - and $y$ - velocities of vehicle which together with sea current velocities lead vehicle along desired course $v$, in the velocities the deviation $\beta$ is not considered,

$V_{c, C P}^{x}, V_{c, C P}^{y}-x$ - and $y$-components of the velocity $V_{c}$, estimated by CP,

$\vartheta$ - a function transforming course $v$ to the range $(0,90)$,

$v$-direct course from a given position of vehicle to waypoint, which does not correspond to the vehicle course $\alpha$,

$V_{a, C P}$ - vehicle velocity magnitude after $\mathrm{CP}$, which is defined as follows:

$V_{a, C P}=\left(\operatorname{getVelocityX}\left(\left|V_{\varepsilon, C P}\right|, \beta_{C P}\right)\right)^{2}+\left(V_{a}+\text { getVelocity }\left(\left|V_{\theta, C P}\right|, \beta_{C P}\right)\right)^{2}$

2 The system of Eq.(22), (23) is used only for $v \notin\{0,90,180,270\}$, e.g. for $v=0: V_{a}^{x}=-V_{c, c p}^{x}$ and $V_{a}^{y}=\left(V_{a}\right)^{2}-\left(V_{a}^{x}\right)^{2}$ where:

getVelocity $X(W, Z)$ and $\operatorname{getVelocity} Y(W, Z)$ are functions which produce $x$ - and $y$ - components of a vector determined by the length $W$ and the direction $Z \in\langle-180,180\rangle$.

The system of Eq.(22), (23) has four different solutions of $V_{a}^{x}, V_{a}^{y}$, in total. The correct solution is the one which leads the vehicle along the course $v$ with the highest resulting velocity. After determining the correct values of $V_{a}^{x}, V_{a}^{y}$, the vehicle course is calculated according to Eq.(25), and then improved according to Eq. (26):

$$
\alpha=\operatorname{getCourse} 360\left(V_{a}^{x}, V_{a}^{y}\right)
$$

$$
\alpha=\alpha-\text { getCourse } 180\left(U_{1}, U_{2}\right)
$$

where:

$$
\begin{aligned}
& U_{1}=\text { getVelocity } X\left(\left|V_{\theta, C P}\right|, \beta_{C P}\right), \\
& U_{2}=\text { getVelocity } Y\left(\left|V_{\theta, C P}\right|, \beta_{C P}\right),
\end{aligned}
$$

and getCourse $180\left(V_{x}, V_{y}\right)$ is a function which determines direction of the vector $V$ expressed in the range $\langle-180,180\rangle$ deg, $V_{x}$ and $V_{y}$ are $x$-and $y$ - components of $V$.

In the case of CP2, another approach is used. Since in case of Eq. (22), (23) it is necessary to know $V_{c, C P}^{x}, V_{c, C P}^{y}$ to determine $\alpha$ which , according to Eq.(21), cannot be estimated as long as $\alpha$ is unknown, CP2 is performed in three phases to fix course of the vehicle. First, Eq.(22), (23) are used to estimate $V_{c, C P}^{x}, V_{c, C P}^{y}$ for $\alpha=45 \mathrm{deg}$, then Eq.(21) is applied to improve the estimations, and finally, procedure of solving Eq. (22), (23) is run again to fix final value of $\alpha$.

\section{EXPERIMENTS}

\section{CONDITIONS OF EXPERIMENTS}

To test what improvement of position accuracy can be achieved when using CP, simulation experiments were carried out. In the experiments, motion of the vehicle was calculated in three different ways. The pattern motion to be used as a point of reference for DRNS was computed as follows:

$$
\begin{gathered}
S_{x, j, G P S}=\sum_{i=1}^{N}\left(V_{a, j}^{x}+V_{\theta, j}^{x}+V_{c, i}^{x}+V_{r, j, i}^{x}\right) t \\
S_{y, j, G P S}=\sum_{i=1}^{N}\left(V_{a, j}^{x} \tan \left(\psi\left(\alpha_{j}\right)\right)+V_{s, j}^{x} \tan \left(\psi\left(\alpha_{j}+\beta\right)\right)+V_{c, i}^{y}+V_{r, j, i}^{y}\right) t
\end{gathered}
$$

where :

$i$ - i-th time step during motion toward $\mathrm{j}$-th way-point;

$i$ - number of way-point;

$t$ - time interval;

$N$ - number of steps necessary to reach way-point;

$V_{r}^{x}, V_{r}^{y}-$ random values such that $\left|V_{r}\right| \in\left\langle 0, r_{\max }\right\rangle$, direction of $V_{r}$ ranges $\langle 0,359\rangle$. 
Eq. (27), (28), reflect determinism of the vehicle and randomness of the environment, the velocities $V_{a}, V_{e}$ representing the vehicle are constant during each run of simulation, whereas, $V_{c}$, and $V_{r}$ are random variables. It is assumed that the vehicle is a deterministic device with a known desired speed and course represented by $V_{a}$ and with an unknown deterministic working inaccuracy represented by $V_{e}$. On the other hand, the environment is random and partly unpredictable which is reflected in $V_{c}$ and $V_{r}$.

$V_{c}$ represents mainly the sea current and wind, so, environmental forces whose characteristic is, on the one hand, of a random nature, but on the other hand, it determines a dominant direction $\alpha_{c}$ and magnitude $\left|V_{c}\right|$ which are parameters of simulation. Value of $V_{c, i}$ is fixed in two stages. First, magnitude of $V_{c}$ is adjusted to the vehicle course $\alpha_{j}$ and the deviation from the course $\beta$; and the following formula is used for this purpose:

$\left|V_{c, i, \alpha_{j}+\beta}\right|=\left|V_{c}\right| \varrho_{s}\left(\operatorname{diffCourse}\left(\alpha_{j}+\beta, \alpha_{c}\right)\right) / \varrho_{s}\left(\alpha_{c}\right)$

where $\varrho_{s}$ is variant of the function $\varrho$ which is symmetrical only with respect to axis $Y$ of the vehicle, which means that $V_{c, i}$ is the same, e.g. for 10 and -10 (or 350) deg, and different at the same time for 10 and 170 degrees. The consequence is that $\varrho_{s}$ more exactly reflects the influence of the sea current on vehicle hull than $\varrho$.

In the second stage, both the direction and magnitude of $V_{c, i}$ are randomly deviated according to the normal distribution with parameters $N\left(\left|V_{c, i, \alpha_{j}+\beta}\right|, \sigma_{V}^{2}\right)$, and $N\left(\alpha_{c}, \sigma_{\sim}^{2}\right)$.

The same deviation is used for the number $N_{d}$ of steps $i$, where $N_{f}$ is a random variable uniformly distributed within $\left\langle 1, N_{d, \max }\right\rangle$ range.

In turn, $V_{r}$ represents all other possible environmental random factors that can affect motion of the vehicle and for that reason it is completely chaotic. Direction of $V_{m}$ is uniformly distributed within $\langle 0,359\rangle$ range, whereas $\left|V_{r}\right|$ is a random uniform value in the range of $\left\langle 0, r_{\max }\right\rangle$, where $r_{\max }$ is a parameter of simulation which is a fraction of $V_{a}$.

The estimated motion without $\mathrm{CP}$ was determined in the following way:

$$
\begin{gathered}
S_{x, j, n \circ C P}=\sum_{i=1}^{N} V_{a, j}^{x} t \\
S_{y, j, n \circ C P}=\sum_{i=1}^{N} V_{a, j}^{x} \tan \left(\psi\left(\alpha_{j}\right)\right) t
\end{gathered}
$$

which means that $S_{\text {nocp }}$ is calculated on the basis of only a desired vehicle velocity $\left|V_{a}\right|$ and course $\alpha$, which are provided to the vehicle course controller and the vehicle drive.

The estimated motion after CP looks, in turn, as follows:

$$
S_{x, j, C P}=\sum_{i=1}^{N}\left(V_{a, j}^{x}+V_{\theta, j, C P}^{x}+V_{c, C P}^{x}\right) t
$$

$S_{y, j, C P}=\sum_{i=1}^{N}\left(V_{a, j}^{x} \tan \left(\psi\left(\alpha_{j}\right)\right)+V_{\varepsilon, j, C P}^{x} \tan \left(\psi\left(\alpha_{j}+\beta_{C P}\right)\right)+V_{c, C P}^{y}\right) t$

where $\left|V_{\theta, C P}^{x}\right|, \beta_{C P}, V_{c, C P}^{x}$, and $V_{c, C P}^{y}$ are the result of applying CP for $\Delta S_{x, i}=S_{x, j, n o C P}-S_{x, j, G P S}$, and $\Delta S_{y, j}=S_{y, j, \text { noCP }}-S_{y, j, G P, j}=1,2$. In all the simulations, the assumption was made that $\beta \in\langle-90,90\rangle$. In consequence, to consider the fact that $V_{\theta}$ can increase or decrease progressive speed of the vehicle, $V_{\theta}$ must be able to change sign, i.e. to be positive or negative. To determine sign of $V_{\theta, C P}$, which together with $\left|V_{\theta, C P}\right|$ and $\beta_{C P}$ is necessary to perform CP, the following simple formula was used:

$$
\operatorname{sign}\left(V_{\theta, C P}^{x}, V_{\theta, C P}^{y}, \alpha\right)=\left\{\begin{array}{l}
(+) \text { if } U_{3}<90 \\
(-) \text { otherwise }
\end{array}\right.
$$

where :

$U_{3}=\operatorname{diffCourse}\left(\right.$ getCours $\left.360\left(V_{\theta, C P}^{x}, V_{\theta, C P}^{y}\right), \alpha\right)$.

After the CP simulations additional tests were also carried out to verify effectiveness of the method for determining vehicle course in conditions of presence of forces which can affect vehicle movement (Section 3.5). Conditions of the tests were the same as those described above. The only difference was that $\$ \backslash$ alpha $\$$ ? was not the course to way-point but the course calculated according to Eq. (22)-(26).

In order to test CP1, CP2 and their influence on accuracy of the DRNS, a virtual vehicle with the motion model defined by Eq. (27), (28) performed thirty missions for each type of $\mathrm{CP}$ and each parameter setting. In each mission the vehicle was moved along a trajectory including 3 way-points. The distance between the way-points was always equal to $100 \mathrm{~m}$, whereas the course between them was completely random for $\mathrm{CP} 1$ and partly random for CP2. In the former case, course to the first way-point was equal to $45 \mathrm{deg}$, whereas to the second one $225 \mathrm{deg}$. Regardless of the correction method being tested, the vehicle, when moving to the first of the two way-points, used DRNS which operated according to Eq. (30), (31). Once the vehicle approached the second way-point it performed the $\mathrm{CP}$ and changed DRNS operation to that based on Eq.(32), (33).

The characteristics of CP1 is two-phase determination of $\left|V_{\theta, C P}\right|, \beta_{C P}$, sign of $V_{\theta, C P}, V_{c, C P}^{x}$, and $V_{c, C P}^{y}$. In the first phase, $\beta_{C P}$ was assumed to be zero and the remaining parameters were fixed according to Eq. (7)-(10). Afterwards, in the second phase, the gradient descent of the function (12) was applied to determine all the parameters. Initial values for gradient descent were the ones calculated in the first phase and, additionally, a value of $\beta_{C P}=-\beta_{C P}^{\max },-\beta_{C P}^{\max }+1, \ldots, 0, \ldots, \beta_{C P}^{\max }-1, \beta_{C P}^{\max }$.

CP2 was also performed in two phases: first, Eq. (17)(20) was used to determine vehicle inaccuracies - both in velocity direction and magnitude, and to estimate direction and magnitude of environmental forces, then Eq. (21) was applied to tune the before estimated magnitude.

In addition to the vehicle trajectory, individual missions differed also in parameter setting. The following parameter values were applied: $t=0.001 \mathrm{~s}, V_{a}=1$ 
$\mathrm{m} / \mathrm{s}, V_{s}=\{-0.3,-0.1,0.1,0.3\} \mathrm{m} / \mathrm{s}, \beta=\{-20,-5,5,20\}$ deg, $\alpha_{c}=20 \mathrm{deg},\left|V_{c}\right|=\{0.1,0.5\} \mathrm{m} / \mathrm{s}, r_{\max }=0.3 \mathrm{~m} / \mathrm{s}$, $\beta_{C P}^{\max }=20 \mathrm{deg}, \sigma_{V}^{2}=0.02, \sigma_{\alpha}^{2}=10, N_{d, \max }=10000$.

In the experiments the assumption was made that vehicle inaccuracies cannot be very high due to application of course and depth controllers. For this reason, the tested values of $\beta$ were not greater than \pm 20 deg, whereas, the magnitude of $V_{\varepsilon}$ was at the most equal to $\pm 0.3 \mathrm{~m} / \mathrm{s}$. During all the tests the sea current was assumed to have the same direction, i.e. equal to $\$ 20 \$ \mathrm{deg}$. The magnitude of the current velocity did not exceeded $0.5 \mathrm{~m} / \mathrm{s}$, i.e. only a half of the vehicle velocity. The remaining parameters of the simulation, i.e. parameters which determine their randomness were fixed so that they could not dominate results of the simulation.

\section{RESULTS OF EXPERIMENTS}

Results of all the tests are given in Tab. 1-3 which include only the ones for $r_{\max }=0.3$, and positive values of $\beta$ and $V_{\varepsilon}$. The reason is that simulations with $r_{\max }=0.1$ and negative values of $\beta$ and $V_{e}$ produced very similar outcomes.

Tab. 1. Average errors $[\mathrm{m}]$ occurred along $100 \mathrm{~m}$ distance

\begin{tabular}{|c|c|c|c|c|c|}
\hline \multirow{2}{*}{} & \multicolumn{2}{|c|}{$\left|V_{s}\right|=0.1$} & \multicolumn{2}{c|}{$\left|V_{s}\right|=0.3$} \\
\cline { 2 - 6 } & $\left|V_{c}\right|=0.1$ & $\left|V_{c}\right|=0.5$ & $\left|V_{c}\right|=0.1$ & $\left|V_{c}\right|=0.5$ \\
\hline \multicolumn{2}{|c|}{ without CP } & 12.84 & 47.96 & 31.1 & 43.19 \\
\hline \multirow{2}{*}{$\beta=5$} & $\mathrm{CP} 1$ & 2.58 & 10.9 & 2.45 & 11.4 \\
\cline { 2 - 6 } & $\mathrm{CP} 2$ & 1.17 & 4.68 & 0.93 & 4.42 \\
\hline \multirow{2}{*}{$\beta=20$} & $\mathrm{CP} 1$ & 3.39 & 8.33 & 2.51 & 10.6 \\
\cline { 2 - 6 } & $\mathrm{CP} 2$ & 1.21 & 4.57 & 1.01 & 5.4 \\
\hline
\end{tabular}

Tab. 2. Maximum errors $[\mathrm{m}]$ occurred along $100 \mathrm{~m}$ distance

\begin{tabular}{|c|c|c|c|c|c|}
\hline \multirow{2}{*}{} & \multicolumn{2}{|c|}{$\left|V_{s}\right|=0.1$} & \multicolumn{2}{c|}{$\left|V_{s}\right|=0.3$} \\
\cline { 2 - 6 } & $\left|V_{c}\right|=0.1$ & $\left|V_{c}\right|=0.5$ & $\left|V_{c}\right|=0.1$ & $\left|V_{c}\right|=0.5$ \\
\hline \multicolumn{2}{|c|}{ without CP } & 18.83 & 58.7 & 38.61 & 74.92 \\
\hline \multirow{2}{*}{$\beta=5$} & $\mathrm{CP} 1$ & 6.07 & 35.64 & 15.69 & 28.69 \\
\cline { 2 - 6 } & $\mathrm{CP} 2$ & 2.74 & 11.57 & 1.87 & 10.92 \\
\hline \multirow{2}{*}{$\beta=20$} & $\mathrm{CP} 1$ & 15.89 & 19.51 & 8.77 & 43.12 \\
\cline { 2 - 6 } & $\mathrm{CP} 2$ & 2.24 & 11.04 & 2.49 & 9.46 \\
\hline
\end{tabular}

Tab. 3. Average deviation from desired course, [deg], with and without applying the method specified in Section 3.5

\begin{tabular}{|c|c|c|c|c|c|}
\hline \multirow{2}{*}{} & \multicolumn{2}{|c|}{$\left|V_{\varepsilon}\right|=0.1$} & \multicolumn{2}{c|}{$\left|V_{\varepsilon}\right|=0.3$} \\
\cline { 3 - 6 } & $\left|V_{c}\right|=0.1$ & $\left|V_{c}\right|=0.5$ & $\left|V_{c}\right|=0.1$ & $\left|V_{c}\right|=0.5$ \\
\hline \multicolumn{2}{|c|}{ without CP } & 4.77 & 13.29 & 4.68 & 16.52 \\
\hline \multirow{2}{*}{$\beta=5$} & $\mathrm{CP} 1$ & 0.73 & 2.92 & 0.65 & 3.45 \\
\cline { 2 - 6 } & $\mathrm{CP} 2$ & 0.46 & 2.31 & 0.25 & 2.4 \\
\hline \multirow{2}{*}{$\beta=20$} & $\mathrm{CP} 1$ & 0.99 & 2.7 & 0.63 & 4.04 \\
\cline { 2 - 6 } & $\mathrm{CP} 2$ & 0.48 & 3.34 & 0.29 & 2.2 \\
\hline
\end{tabular}

Generally, as expected, the simulations showed that CP added to the DRNS improves its accuracy, moreover, in some cases the improved accuracy was even thirty-fold greater (Tab. 1, the result for $\mathrm{CP} 2, \beta=5,\left|V_{g}\right|=0.3,\left|V_{c}\right|=0.1$ ). Tab. 2 shows that in conditions with a stronger influence of the environment $\left(\left|V_{c}\right|=0.5\right)$ the neglecting of correction may even result in the error of $70 \mathrm{~m}$ in value which is almost the whole distance covered by the vehicle between way-points. This means that CP embedded in DRNS is a necessary condition for applying BAUV at sea. When the vehicle is not equipped with such devices like log which can provide more precise navigational information mainly about velocity of the sea current, $\mathrm{CP}$ can be the only way to make the vehicle capable of operating at sea. Relying only on resetting position of the vehicle to GPS position, when only it is available, seems to be definitely insufficient, particularly when distance between way-points is large. In such case, safety of the vehicle would require it to appear very frequently on the surface to record GPS position. The CP enables the vehicle to cover longer distances beneath the surface, without necessity to frequently correct the position by means of GPS.

When comparing $\mathrm{CP} 1$ and $\mathrm{CP} 2$ it is clear that $\mathrm{CP} 2$ outperforms $\mathrm{CP} 1$ as in all cases positions corrected by $\mathrm{CP} 2$ are more accurate than those obtained with CP1. Of course, it is not a surprising result which is mainly due to the fact assumed in CP1 that the environmental forces are constant regardless of mutual arrangement of velocity vectors representing the vehicle and the environment. However, in spite of the fact that $\mathrm{CP} 2$ outperforms $\mathrm{CP} 1$, the influence of the latter on position accuracy is definitely positive compared to the case without CP. In this case (except of that with $V_{c}=0.1$ and $\left.V_{e}=0.3\right)$ the average improvement of the accuracy is approximately four-fold greater. Generally, the results of CP1 mean that at the position error not exceeding $100 \mathrm{~m}$ in the worst case, the vehicle can cover a bit greater distance than $200 \mathrm{~m}$ (the maximum error for $\mathrm{CP} 1=43.12 \mathrm{~m}$ ), however, in the average case, it is almost $1000 \mathrm{~m}$ distance covered without appearing on the surface on the assumption that the sea current is invariable on the distance.

The results of CP2 are the most promising. As mentioned above, in this case, the largest average improvement of position accuracy is even thirty-fold greater compared to situation without CP. In the remaining cases, it is more or less tenfold greater with the effect that when the position error not to exceed $100 \mathrm{~m}$ in the worst case, the vehicle can cover almost $1000 \mathrm{~m}$ distance, however, in the average case, it is as long as $2000 \mathrm{~m}$ voyage without emerging. As previously, such result is achievable provided that the sea current is invariable in vehicle operational area.

Tab. 3 shows results of the additional simulations whose goal was to test how information from the $\mathrm{CP}$ can improve quality of vehicle path to a way-point. Typically, the vehicle course corresponds to a direct course to the way--point, which under the conditions of the sea current, wind, and inaccuracies coming from the vehicle itself, may result in a path very far from the optimum one. However, when DRNS is supplied with additional information about possible sources of inaccuracies it can use this information to fix the course of the vehicle in a more optimum manner e.g. that presented in Section 3.5.

The simulation results given in Tab. 3 showed to what extend application of the information from $\mathrm{CP}$ can smooth out the vehicle path. At small influence of external factors, 
the improvement of vehicle course compared to the optimum one, i.e. the course leading vehicle directly to a way-point, is almost invisible. However for $V_{c}=0.5$ the improvement is even eight-fold greater.

\section{SUMMARY}

Underwater navigation of underwater vehicles without devices which provide precise navigational information about course and velocity with respect to Earth is a highly challenging problem which particularly affects small underwater units unable, because of their size, to carry large navigational instruments. One solution of the problem is the use of Simultaneous Localization and Mapping (SLAM), however, it requires moving the vehicle over a short distance from the sea bottom in order to refer position of the vehicle to position of visible landmarks.

When navigation close to the bottom is impossible the only method which can be applied to improve precision of underwater navigation is estimation of all unknown forces having influence on vehicle motion and taking them into account during calculations. This paper presented two solutions which can be applied for that purpose. In them it is assumed that two factors can affect all inaccuracies which can appear during underwater navigation, namely, external factors like wind and sea current which are jointly called environmental factors, and vehicle internal factors such as inaccuracies caused by lack of symmetry in vehicle drive, systematic compass errors etc. To estimate their influence on vehicle motion, GPS position has to be recorded from time to time and position error to be determined. The problem is, however, what position accuracy can be achieved by applying estimates fixed in this manner. Answer to this question is a very important issue because it may decide about distance which the vehicle can safely cover before it has to rise to the surface for taking GPS position.

The presented paper which is the report on simulation tests, offers the first step to answering that question. The tests revealed that estimation methods presented in the paper can significantly improve operation of underwater navigation. The results of the simulation showed that , under stable conditions of the sea current and wind, the vehicle can safely move beneath the surface over the distance as long as $1000 \mathrm{~m}$ and even $2000 \mathrm{~m}$ - in favorable conditions.

Of course, this result is based on the rather unrealistic assumption that the sea current and other environmental forces are invariable over greater areas of the sea. In the performed simulations the current could change both the direction and magnitude, however, the changes incorporated into the sea current model, introduced only slight random perturbation characteristics to the current with stable parameters. Unfortunately, lack of any device for measuring parameters of external forces affecting motion of the vehicle makes it impossible to effectively counteract the forces of variable nature. However, we always know where the vehicle has to operate and thereby we also know properties of the future operational area with the effect that we can appropriately adjust its navigational system to the operational area in question. Variability of the sea current in a considered area results in necessity of more frequent emerging onto the surface, whereas, the opposite situation leads to a longer distance which the vehicle can cover underwater.

The other problem is reliability of course information acquired from different sorts of compasses as well as accessibility of reliable GPS signal for supporting the dead reckoning navigation. Contemporary digital compasses even the cheapest ones, if appropriately mounted far away from all sources of magnetic field, seem to be sufficiently accurate and thereby they should not have considerable negative influence on quality of navigational system. Unavailability of GPS signal is a more serious problem. When the vehicle operates in GPS-denied or GPS-inhibited environment, it is necessary to provide it with other source of pattern navigational information to reduce position error of the dead reckoning. One solution can be e.g. an optical system with the ability of identifying landmarks visible on the coast and to use them to estimate the vehicle position. Unfortunately, according to the knowledge of this author, such solution is still an undiscovered area in the marine technology, remaining therefore an attractive field for further research

\section{BIBLIOGRAPHY}

1. B. Allotta, A. Caiti, L. Chisci, R. Costanzi, F. Di Corato, C. Fantacci, D. Fenucci, E. Meli, A. Ridolfi : Development of a Navigation Algorithm for Autonomous Underwater Vehicles. IFAC-PapersOnLine, Vol. 48, Is. 2, 2015, pp. 64-69

2. S. Arulampalam, S. Maskell, N. Gordon and T. Clapp: A tutorial on particle filters for on-line non-linear/nonGaussian Bayesian tracking. IEEE Trans, Signal Processing, 50 (2), 2002, pp. 174-188

3. D. Li, D. Ji, J. Liu, Y. Lin : A Multi-Model EKF Integrated Navigation Algorithm for Deep Water AUV. International Journal of Advanced Robotic Systems, Vol. 13, Is. 1, 2016

4. A. Doucet, N. de Freitas, N. Gordon: Sequential Monte Carlo methods in practice. Statistics for Engineering and Information Science. Springer-Verlag, New York, 2001

5. G. Einicke, L. White: Robust Extended Kalman Filtering. IEEE Trans. Signal Processing. 47 (9), 1999, pp. 2596-2599

6. H. Johnnsson, M. Kaess, B. Englot, F. Hover, JJ. Leonard: Imaging Sonar-Aided Navigation for Autonomous Underwater Harbor Surveillance. 2010 IEEE/RSJ International Conference on Intelligent Robots and Systems, 2010, pp. $4396-4403$

7. S. Julier, J. Uhlmann : Unscented filtering and nonlinear estimation. Proc. of the IEEE, 92, 2004, pp. 401-422. 
8. J. Kinsey, R. Eustice, L. Whitcomb: A survey of underwater vehicle navigation: recent advances and new challenges. In Proc. Conf. Maneuvering Control Marine Craft. 2006, pp. 1-12

9. T. Leszczynski : The effect of interference parameters on the exploitation capabilities of an underwater vehicle. Scientific Journal of Polish Naval Academy, 3(26), 2016, pp. 85-106

10. L. Paull, S. Saeedi, M. Seto, H. Li : AUV Navigation and Localization: A Review. IEEE Journal of Oceanic Engineering, Volume: 39, Issue: 1, 2014, pp. 131 - 149

11. M. Malec, M. Morawski, and J. Zając: Fish-like swimming prototype of mobile underwater robot. Journal of Automation, Mobile Robotics and Intelligent Systems, Vol. 4, No. 3, 2010, pp. 25-30

12. A. Martinez, L. Hernandez, H. Sahli, Y. Valeriano-Medina, M. Orozco-Monteagudo, D. Garcia-Garcia : ModelAided Navigation with Sea Current Estimation for an Autonomous Underwater Vehicle. International Journal of Advanced Robotic Systems, Vol.12, Is. 7, DOI: https:// doi.org/10.5772/60415

13. T. Praczyk : A quick algorithm for planning a path for biomimetic autonomous underwater vehicle. Scientific Journals of Maritime University of Szczecin, No. 45 (117), 2016, pp. 23-28

14. T. Praczyk : Using Genetic Algorithms for Optimizing Algorithmic Control System of Biomimetic Underwater Vehicle. Computational Methods in Science and Technology (CMST), Vol. 21 (4) 2015, pp. 251-260

15. R. Siegwart, I. R. Nourbakhsh : Introduction to Autonomous Mobile Robots. MIT Press, Cambridge 2004

16. P. Szymak, T. Praczyk, K. Naus, M. Malec, M. Morawski : Research on biomimetic underwater vehicles for underwater ISR. Ground/Air Multisensor Interoperability, Integration, and Networking for Persistent ISR VII, edited by Michael A. Kolodny, Tien Pham, Proc. of SPIE Vol. 9831, 98310K

17. P. Szymak, M. Malec, M. Morawski : Directions of development of underwater vehicle with undulating propulsion. Polish Journal of Environmental Studies, Hard Publishing Company, Vol.19, No. 3, Olsztyn 2010, pp. $107-110$

18. E. Wan, R. Merwe: The Unscented Kalman Filter. T. Haykin Edition, New York, NY, USA, 2001

19. W. Zeng, L. Wan, T. Zhang : Simultaneous localization and mapping of autonomous underwater vehicle using looking forward sonar. Journal of Shanghai Jiaotong University (Science), Vol. 17, 2012 , Is. 1, pp 91-97,
20. T. Zhang, W. Zeng, and L. Wan : Underwater simultaneous localization and mapping based on forward--looking sonar. Journal of Marine Science and Application, (2011) pp.10:371. doi:10.1007/s11804-011-1082-1

21. http://cmtm.pg.gda.pl/systemy-techniki-glebinowej

\section{CONTACT WITH THE AUTHOR}

\author{
Tomasz Praczyk \\ e-mail: t.praczyk@amw.gdynia.pl \\ Polish Naval Academy \\ Institute of Naval Weapon \\ Śmidowicza 69, 81-127 Gdynia \\ Poland
}

